
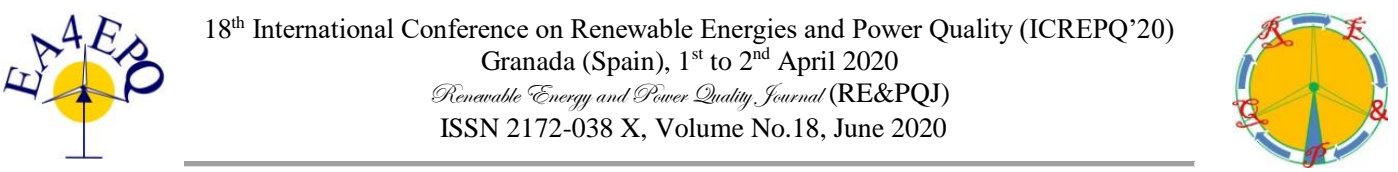

\title{
Control Optimization of the Offshore HVDC Grid based on Modular Multilevel Converter for Improving DC Voltage Stability
}

\author{
Atousa Elahidoost ${ }^{1}$, Elisabetta Tedeschi ${ }^{2}$ \\ Department of Electric Power Engineering \\ Norwegian University of Science and Technology (NTNU) \\ Trondheim, Norway \\ Phone/Fax number: +0047 73595000, E-mail: atousa.elahidoost@ntnu.no', elisabetta.tedeschi@ntnu.no²
}

\begin{abstract}
The aim of this paper is to reduce the voltage oscillation of the HVDC cable in an offshore point-to-point configuration based on the modular multilevel converter. An optimization methodology based on semidefinite programming is proposed, which improves the DC voltage stability under the worst-case initial perturbation scenario. This goal is achieved by implementing a centralized optimal linear feedback controller, which guarantees Lyapunov stability margins while considering constraints on control inputs and state variables. The scope of this work is twofold. First, to analyze small-signal eigenvalue stability of the modular multilevel converter-based HVDC grid to identify the source of the oscillations. Second, to use this analysis as a basis to design a centralized optimal linear feedback controller to improve stability margins. The linear feedback controller is first applied as an additional control layer to the standard control of the offshore point-to-point HVDC grid, and then it is implemented to substitute the droop control function of the grid. Finally, the applicability and validity of the results are verified by time-domain simulations.
\end{abstract}

Keywords. DC voltage stability, Lyapunov stability, modular multilevel converter, offshore HVDC grid, centralized optimal linear feedback controller.

\section{Nomenclature}

$\begin{array}{ll}R_{g}, L_{g} & \text { Equivalent grid-side resistance and inductance } \\ R_{a}, L_{a} & \text { MMC arm resistance and inductance } \\ C_{S M} & \text { MMC submodule capacitance } \\ R_{e q}, L_{e q} & \text { MMC equivalent resistance and inductance defined } \\ & \text { as } R_{e q}=R_{g}+R_{a} / 2, L_{e q}=L_{g}+L_{a} / 2 \\ C_{e q} & \text { MMC equivalent capacitance defined as } \\ & \begin{array}{l}C_{e q}=C_{S M} / N \\ C_{d c}\end{array} \quad \text { Equivalent DC-side capacitance } \\ R_{c}, L_{c}, C_{c} & \text { Equivalent HVDC cable resistance, inductance, and } \\ N & \text { capacitance } \\ N & \text { Number of MMC submodules in each arm } \\ n_{u, l} & \text { MMC upper, and lower arm insertion indices } \\ v_{g} & \text { Equivalent grid-side voltage } \\ v_{v}, i_{v} & \text { MMC AC-side voltage and current } \\ v_{c c}, i_{c c} & \text { MMC circulating voltage and current } \\ v_{u l,}, i_{u, l} & \text { MMC upper and lower arm voltage and current } \\ v_{d c}, i_{d c} & \text { DC-side voltage and current } \\ i_{c} & \text { Equivalent HVDC cable current } \\ P_{a c}, Q_{a c} & \text { AC-side active and reactive power } \\ w_{\sum, z} & \text { Zero sequence energy sum } \\ w_{\Sigma, z, f f} & \text { Zero sequence energy sum feedforward } \\ * & \text { Reference value in control system } \\ \mathrm{X} & \text { X }=x_{d}+j x_{q} \\ s & \text { Scalar quantity where } s>0\end{array}$
$M$
$Q, Y$
Weight matrix where $M=\hat{M} \hat{M}^{T} \geq 0$
Unique matrices where $Q>0$ and $Y>0$

\section{Introduction}

Offshore wind farms as renewable sources of energy in the North Sea are a promising solution to overcome the increasing energy demand and the adverse impact of global warming. High Voltage Direct Current (HVDC) cables together with Voltage Source Converters (VSC) are the common technology for large-scale offshore wind energy transportation [1]. The preferred VSC for this application is the Modular Multilevel Converter (MMC) due to the modularity and scalability, high-energy efficiency and high quality of the produced current and voltage waveforms [2]. A point-to-point connection is the conventional configuration for the existing and planned offshore HVDC wind farms [3]. However, it is anticipated that point-to-point connections will gradually evolve into multi-terminal and eventually meshed networks in the North Sea as a prerequisite of $100 \%$ renewable European smart grid [4]. The interconnection of the independent offshore HVDC wind farms with different technologies introduces various control and stability challenges. Optimization strategies are required to address these technical obstacles in the grid transmission expansion planning (TEP). However, to the best of our knowledge, there are very few works, which investigate the TEP problem in hybrid AC/DC grids from the control and stability point of view in conjunction with the cost and power loss minimization [5-7]. Since DC voltage stability is of paramount importance in multiterminal HVDC grids [8, 9], a method for reducing DC voltage oscillation as the main objective in HVDC grid expansion problem is proposed in [6,7]. However, the converter topology used in $[6,7]$ is the 2-level VSC, which in recent HVDC applications is increasingly being replaced by MMC technology.

This paper investigates whether the optimization and control methodology previously introduced in $[6,7]$ for DC voltage stability enhancement in multi-terminal HVDC grids could still be valid in a more realistic case with MMCs as the converter of choice. In other words, the goal is to find out if the dynamics and control parameters of the converter, which are specific to MMCs could contribute to DC voltage instability under the 
worst-case perturbation scenario, and in such case if it can be mitigated by appropriate control. Therefore, an MMCbased point-to-point offshore HVDC grid is modeled as the reference case. First, the state-space model of the grid is developed, which is the basis for both small-signal eigenvalue stability analysis and optimization procedure. The small-signal eigenvalue stability analysis is performed to gain a better insight into the possible instability sources in the grid. The main contribution of the paper is then to design a Centralized Optimal Linear Feedback Controller (COLFC), which can improve grid stability margins by reducing DC voltage oscillation under the worst-case initial perturbation scenario. The COLFC can be implemented either as an additional control layer or as a droop controller. Finally, the performance of the COLFC is compared with the conventional droop controller through time-domain simulation.

\section{Steady-State Time-Invariant State-Space Model of the Offshore Point-to-Point HVDC Grid}

The reference model configuration used in this paper is presented in Fig.1 while the values are extracted from [10]. In this section, the Steady-State Time-Invariant (SSTI) state-space representation of the grid consisting of MMCs and an HVDC cable is demonstrated. The statespace model is derived as a prerequisite for small-signal eigenvalue stability analysis and the control optimization process.

\section{A. MMC SSTI Modeling and Control}

The SSTI equations of the MMC are derived based on the simplified zero-sequence model with reduced order [11]. This model only considers the zero-sequence components of the MMC arm capacitive energy sum and circulating current, and ignores the $d q$-components due to their relatively small impact under the assumed modulation strategy. Since the reduced-order model preserves a sufficient degree of accuracy despite having a lower number of state-variables, it becomes an attractive alternative for performing large-system stability studies, and its sufficient fidelity has been proved in previous literature [11]. An overview of the converter configuration and control block diagrams is shown in Fig.2 and Fig.3. The energy-based modeling approach with compensated modulation (CM) [11] is used to represent the MMC internal current and energy dynamics. As a result of CM control, the insertion indices compensate for the arm voltage oscillations. For the sake of completeness, a summary of the reduced-order SSTI MMC equations under CM is given as follows.

$$
\frac{d}{d t} i_{v, d q}=\frac{1}{L_{e q}}\left(v_{v, d q}-v_{g, d q}-R_{e q} i_{v, d q}-j \omega L_{e q} i_{v, d q}\right)
$$

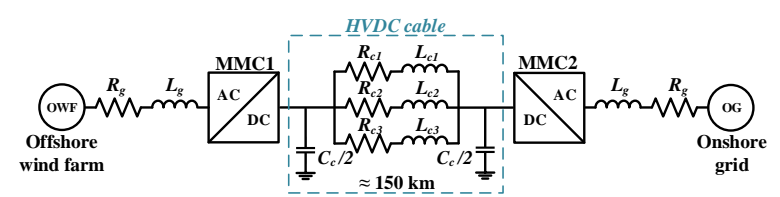

Fig. 1: MMC-based point-to-point offshore HVDC grid.

$$
\begin{aligned}
& \frac{d}{d t} i_{c c, z}=\frac{1}{L_{a}}\left(\frac{v_{d c}}{2}-v_{c c, \mathrm{z}}-R_{a} i_{c c, z}\right) \\
& \frac{d}{d t} v_{d c}=\frac{1}{\left(C_{d c}+\frac{C_{c}}{2}\right)}\left(i_{c}-3 i_{c c, \mathrm{z}}\right) \\
& \frac{d}{d t} w_{\Sigma, z} \approx-\frac{1}{2}\left(v_{v, d}^{*} i_{v, d}+v_{v, q}^{*} i_{v, q}\right)+2 v_{c c, z}^{*} i_{c c, z}
\end{aligned}
$$

The MMC control equations, which are required for the state-space representation of the entire system, can be derived according to the control block diagrams shown in Fig.2 and Fig.3. The commonly applied cascaded control loops are utilized in this application where the outer control loop is composed of active power controller with droop, reactive power controller and zero-sequence energy sum controller. Droop control function is exclusively applied for both offshore and onshore terminals to further investigate the grid expansion potential to the multi-terminal configuration from the stability point of view; although for point-to-point wind farm applications the onshore grid droop coefficient should be set to zero. The outer control loop provides set points for the inner controllers consisting of AC-side current controller and zero-sequence circulating current controller. Modulus optimum and symmetrical optimum strategies are used to tune PI controllers' parameters [12].

\section{B. HVDC Cable SSTI Modeling}

The frequency-dependent cascaded pi-section model with parallel series branches introduced in [1] is used to formulate the offshore HVDC cable as shown in Fig.1. The model is suitable for small-signal eigenvalue stability analysis and allows for state-space representation. Thus, the SSTI HVDC cable equations composed of one pi-section and three parallel branches used in this paper are:

$$
\frac{d}{d t} i_{c, i}=\frac{1}{L_{c, i}}\left(v_{d c 1}-v_{d c 2}-R_{c, i} i_{c, i}\right) \quad i=1,2,3
$$

where the cable capacitor effect, $C_{c}$, is considered in equation (3).

\section{SSTI State-Space Model of the Offshore Point-to- Point HVDC Grid}

The SSTI state-space equation standard form is [13]: $\dot{x}=f(x, u), \quad z=g(x, u)$

where $x, u$ and $z$ are defined as a state vector, control input vector, and output vector respectively.

Therefore, the SSTI state-space representation of the proposed grid given in Fig. 1 is composed of 25 state variables and 8 control inputs as follows:

$$
\begin{aligned}
x(t)= & {\left[i_{v, d 1} i_{v, q 1} \xi_{i v d 1} \xi_{i v q 1} i_{c c, z 1} \xi_{i c c z 1} v_{d c 1} \xi_{P a c 1} \xi_{Q a c 1} \ldots\right.} \\
& w_{\sum, z 1} \xi_{w \sum z 1} i_{c 1} i_{c 2} i_{c 3} i_{v, d 2} i_{v, q 2} \xi_{i v d 2} \xi_{i v q 2} \ldots \\
& \left.i_{c c, z 2} \xi_{i c c z 2} v_{d c 2} \xi_{P a c 2} \xi_{Q a c 2} w_{\sum, z 2} \xi_{w \sum z 2}\right] \\
u(t)= & {\left[v_{d c 1}^{*} P_{a c 1}^{*} Q_{a c 1}^{*} w_{\sum, z 1}^{*} v_{d c 2}^{*} P_{a c 2}^{*} Q_{a c 2}^{*} w_{\sum, z 2}^{*}\right] }
\end{aligned}
$$




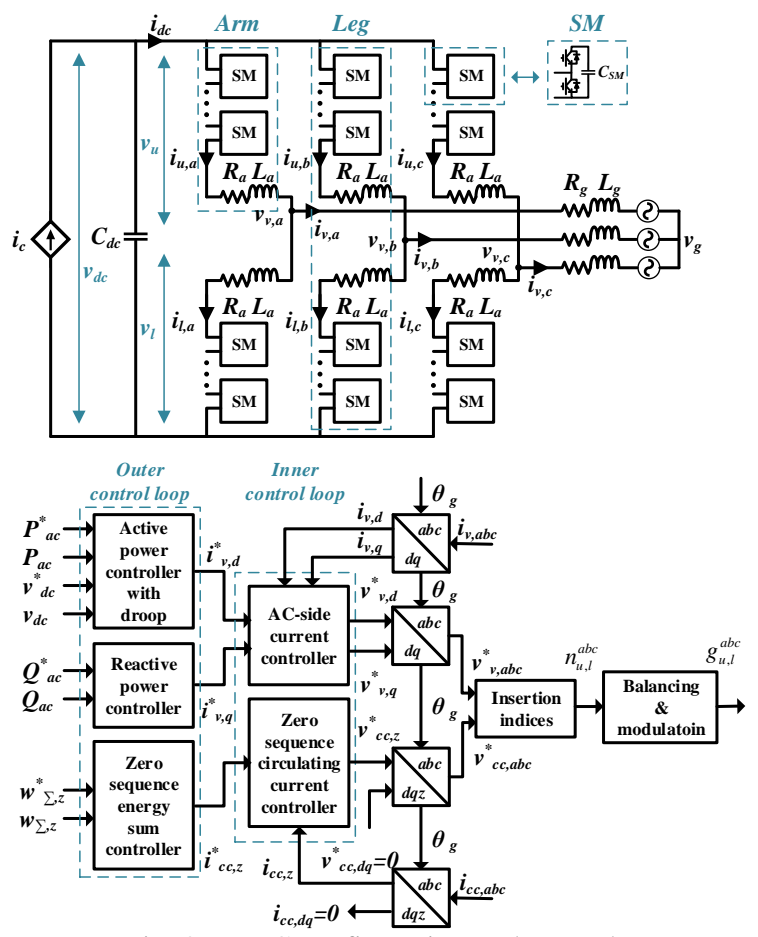

Fig. 3: MMC configuration and control.

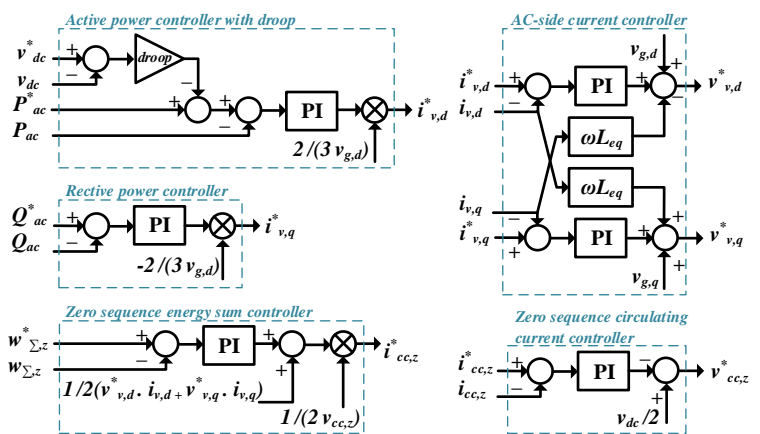

Fig. 2: MMC inner and outer control loop block diagrams.

where the indices 1 and 2 are associated with the MMC1 and MMC2 state variables respectively, and the state variable $\xi$ defines the integrator state of the PI controller for every control loop.

To verify the accuracy of the SSTI model of the proposed grid, its performance is compared through timedomain simulation with a circuit-based model of the grid developed in MATLAB/Simulink with the Simscape toolbox. In the circuit-based model, the Arm Averaged Model (AAM) of the MMC is used, in which each converter arm is modeled as a controlled voltage source including an equivalent arm capacitance [14]. It should be noted that in AAM, the dynamics associated with the submodule (SM) capacitor voltage balancing algorithm and switching operation are ignored. The control strategies in both SSTI and circuit-based models are based on Fig. 2 and Fig. 3.

Dynamics of the grid are excited by applying a $10 \%$ step increase of MMC2 active power reference at $t=1 \mathrm{~s}$ and a $10 \%$ step increase of the MMC2 zero-sequence energy sum at $t=2 \mathrm{~s}$. Due to the droop control operation, an increase in the active power reference is followed by the reduction of the equivalent DC-side voltage. Timedomain simulation results are presented in Fig. 4. DCside voltage, zero-sequence circulating current and zerosequence energy sum waveforms of MMC2 are shown as an example. As can be seen in the figure, both SSTI and circuit-based models are matching with fairly good precision and it can be concluded that the SSTI model is accurate enough to catch MMC dynamics under the transients.

\section{Small-Signal Eigenvalue Stability Analysis of the Offshore Point-to-Point HVDC Grid}

The nonlinear SSTI state-space equations are the prerequisite for small-signal eigenvalue stability analysis. The first step is to solve $\dot{x}=0$ to find the steady-state operating point of the grid. Then, the model can be linearized around the operating point using [13]:

$$
\begin{aligned}
& \Delta \dot{x}=A\left(x_{0}\right) \Delta x+B\left(x_{0}\right) \Delta u \\
& \Delta z=C\left(x_{0}\right) \Delta x+D\left(x_{0}\right) \Delta u
\end{aligned}
$$

where $x_{0}$ is the steady-state operating point of the system.

The matrix $A$ (characteristic matrix), is used to extract the system eigenvalues, which are depicted in Fig. 5. Real and imaginary parts of the eigenvalues can be used to obtain oscillation frequency and damping factor associated with each eigenvalue or mode. Most of the eigenvalues in the reference grid have an imaginary part of zero, which means their oscillation frequency is zero or their damping factor is equal to one. The value of the most dominant eigenvalue or pole that is closest to the right half-plane (RHP) and has a non-zero imaginary part is $\lambda_{i}=-83 \pm j 326$, which corresponds to the oscillation frequency of about $50 \mathrm{~Hz}$. This frequency is the one observed in Fig. 4 and can be a source of instability. Now to identify which state variables contribute to this mode, participation factor analysis is performed. The results are
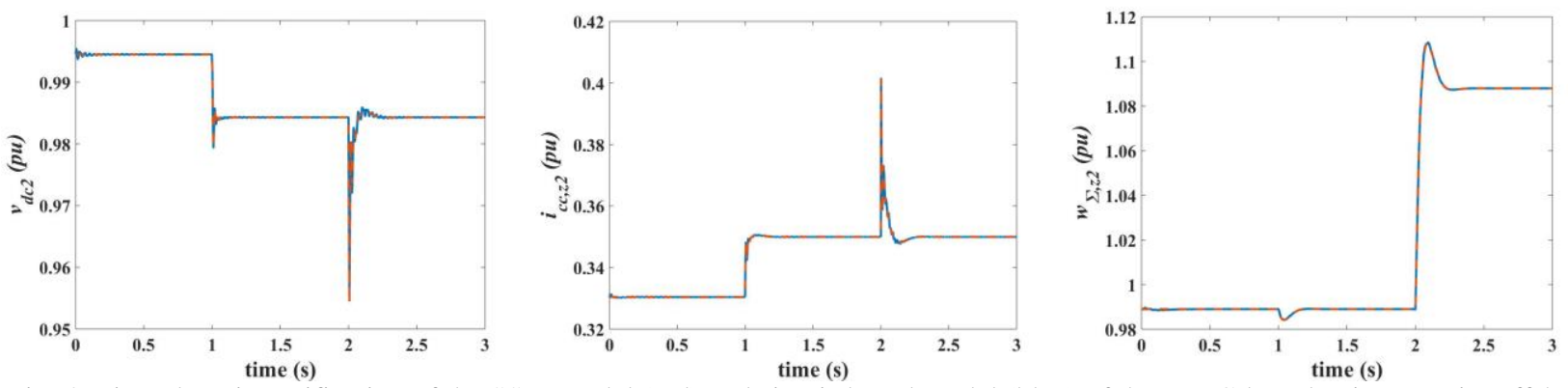

Fig. 4: Time-domain verification of the SSTI model (red) and circuit-based model (blue) of the MMC-based point-to-point offshore HVDC grid. 


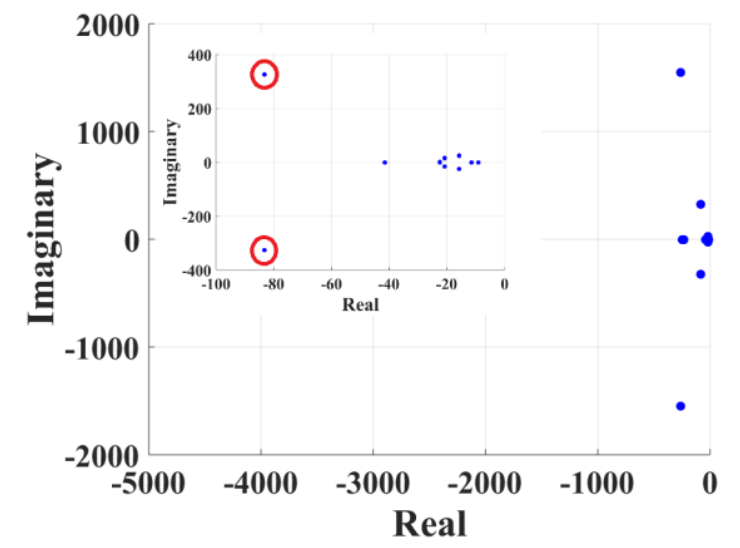

Fig. 5: Offshore point-to-point HVDC grid eigenvalues.

shown in Fig. 6. As can be seen, converters' DC-side voltage, $v_{d c l \& 2}$, and integral parameter of the AC-side

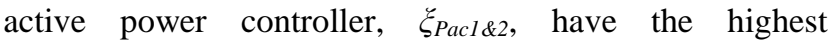
contribution to this mode. These two state variables are somehow related through the droop control function. It is possible to obtain a better understanding of the droop control effect on system stability via eigenvalue trajectory study as shown in Fig. 7. In this study, the droop coefficient of both converters is varied from 0.05 to 0.2 , which is a conventional droop control range. The resulting figure shows that the system becomes unstable at droop = 0.05 and the stability margin improves as the droop coefficient increases. Therefore, it is obvious that the droop control function can play a significant role in DCside voltage stability.

\section{Control Optimization of the Offshore Point-to-Point HVDC Grid}

The optimization technique applied in this paper is based on the Semi Definite Programming (SDP), which is a convex optimization problem and is converted into Linear Matrix Inequality (LMI) formulation. The optimization methodology is inspired from [15] and is adapted into the paper application. It has previously been used to improve DC voltage stability in multi-terminal offshore HVDC grid expansion problems based on 2-level VSC while also considering other criteria such as cost or loss minimization or wind intermittency effect $[6,7]$. The optimization objective in this paper is to reduce DC voltage oscillation under worst-case initial perturbation in the reference grid, which is based on the MMC. The optimization methodology simultaneously finds the worstcase initial perturbation scenario of the system state variables and improves the DC voltage stability margins by proposing a COLFC. COLFC is so flexible that it can both operate as an extra control layer and substitute the droop control function. The optimization algorithm is solved using the YALMIP toolbox [16] of MATLAB in combination with the MOSEK solver [17], and is formulated as follows:

$$
\begin{aligned}
& \frac{1}{J_{o s c i}^{*}}=\max _{s>0, Q>0, Y} s \\
& \text { s.t. } \\
& {\left[\begin{array}{cc}
(A Q+B Y)+(A Q+B Y)^{T} & Q C^{T} \hat{M} \\
\hat{M}^{T} C Q & -I
\end{array}\right] \leq 0} \\
& {\left[\begin{array}{cc}
Q & Y^{T} \\
Y & s E_{u}^{-1}
\end{array}\right] \geq 0} \\
& Q-s E_{x}^{-1} \geq 0
\end{aligned}
$$

where $J_{o s c i} \leq(1 / s)$ is the DC voltage oscillation index to minimized and is defined as:

$$
\begin{aligned}
& J_{o s c i}=\int_{0}^{\infty} z(t)^{T} M z(t) d t \\
& z(t)=C x(t)
\end{aligned}
$$

where $C$ acts as a weight matrix to select a subset of desired state variables, consisting of converters' DC voltage, where the aim is to minimize their oscillation.

Centralized optimal linear feedback controller, $K$, whose structure is depicted in Fig. 8, is equivalent to:

$u(t)=K x(t)$

$Y=K Q$

And $E_{u}>0$ and $E_{x}>0$, are interpreted as:

$$
\begin{aligned}
& u(t) \in U=\left\{\Delta u \in R_{8}: u^{T} E_{u} u \leq 1\right\} \\
& x(0) \in X_{0}=\left\{x \in R_{25}: x^{T} E_{x} x \leq 1\right\}
\end{aligned}
$$

In summary, the optimization objective is implemented through (10a), which is the DC voltage oscillation minimization. Out of three constraints, the first one (10b) is associated with the Lyapunov stability equation, and the second and third one (10c and 10d, respectively) are related to the confinement of the control inputs and state variables in ellipsoidal sets, reflecting their physical limits.

\section{A. COLFC as an Extra Control Layer}

In this section, to investigate the performance of the control optimization methodology in stability improvement, a 20\% step reduction (the reason for this choice is clarified below) is introduced to MMC2 DCside voltage, $v_{d c 2}$. Fig. 9(a) and 9(b) show the simulation results for the time-domain circuit-based model before

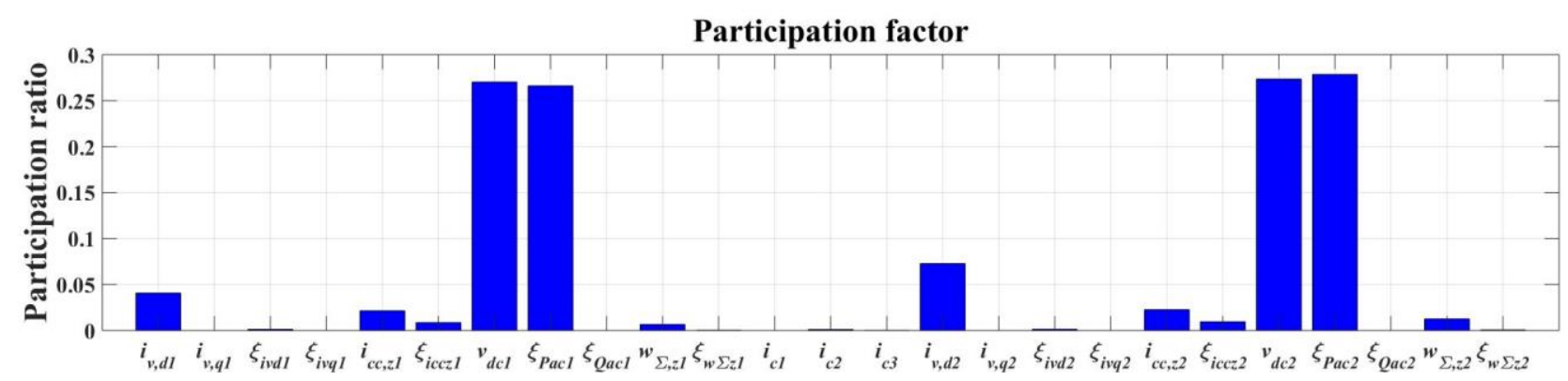

Fig. 6: Participation factor analysis for the mode $\lambda_{i}=-83 \pm j 326$. 


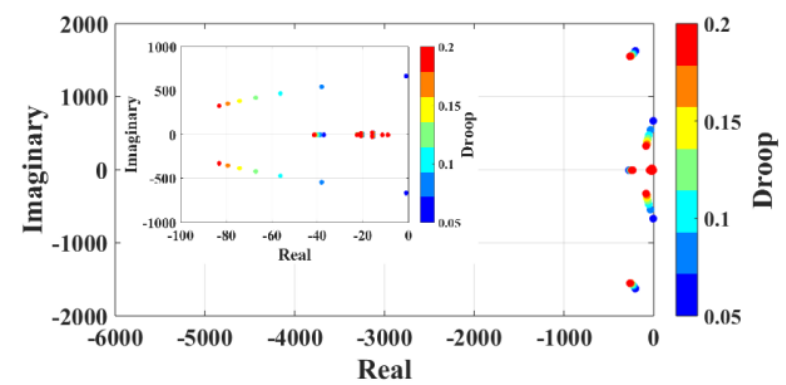

Fig. 7: Eigenvalue trajectory for droop variation from 0.05 to 0.2 .

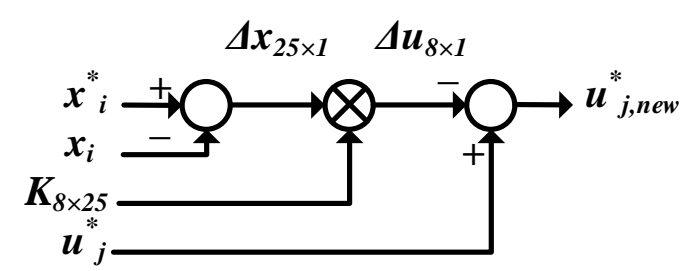

Fig. 8: Centralized optimal linear feedback controller.

and after applying COLFC. As can be seen from Fig. 9(a), $v_{d c 2}$ value is $0.99 \mathrm{pu}$ under the steady-state condition and after the step, it starts to oscillate at around $50 \mathrm{~Hz}$ (related to $\lambda_{i}=-83 \pm j 326$ ) without being damped. In fact, the system has become unstable at the new operating condition because of the eigenvalue move towards the instability region (RHP). By running the optimization code, the worst-case initial perturbation obtained is pointing at $v_{d c l \& 2}$ state variables reduction, which is why a $20 \%$ step reduction of the MMC2 DC-side voltage is chosen as the test-case. Furthermore, Fig. 9(b) shows how the addition of the COLFC has damped the oscillation and made the system stable. The eigenvalue responsible for instability has moved away from the instability region and become equal to $\lambda_{i}=-242 \pm j 404$. Another interesting result is that COLFC is an $8 \times 25$ matrix where the elements with the highest value are the ones involving $v_{d c l \& 2}^{*}$ and $\xi_{\text {Pacl } \& 2}$ and this is exactly in alignment with the results obtained in section 3. From the results, it is clear that COLFC is a centralized optimal controller that can readjust the converters' droop control value to assure system stability under the worst-case initial perturbation scenario.

\section{B. COLFC Substituting the Droop Control Function}

The ability of the COLFC is tested in this section to see whether it can operate as a droop control function (i.e. by replacing the standard droop) or not. Moreover, it is verified if COLFC can perform better than conventional droop control from the stability point of view. The first step is to set the grid droop coefficient from 0.2 to zero. This action makes the system unstable and small-signal eigenvalue stability analysis confirms this by showing an eigenvalue in RHP, which originates from $v_{d c l \& 2}$ instability based on the participation factor analysis. Fig. 9(c) demonstrates $v_{d c 2}$ waveform after applying COLFC with the value of $1.003 \mathrm{pu}$, and $v_{d c l}$ settles at 1.007 pu under steady-state condition (which is not shown in this figure). It is apparent that COLFC is capable of taking the droop control responsibility under the worst-case perturbation scenario, which is again converters' DC-side voltage reduction according to the optimization algorithm. However, COLFC matrix elements with the highest value involved in stabilization in the absence of the conventional droop are $w^{*}{ }_{\Sigma, z l \& 2}$ and $\xi_{\text {Pacl\&2. }}$ To further investigate the COLFC under grid dynamics and transients, a $10 \%$ step increase is introduced to $P_{a c 2}$, which results in $v_{d c 2}$ reduction, and the MMC2 waveforms are depicted in Fig. 9(d), 9(e) and $9(f)$. It can be seen that the grid remains stable with COLFC operation under the dynamics, and the power balance between AC-side, DC-side, and MMC internal energy storage components is preserved. Nevertheless, a comparison of Fig. 9(b) and Fig. 9(d) shows the combination of droop and COLFC has faster oscillation damping than the COLFC alone and it can be verified through the DC voltage oscillation index value. The oscillation index has a lower value (21 versus 123$)$ in the case of COLFC as an extra control layer.

\section{Conclusion}

In this paper, a method for control optimization of the MMC-based point-to-point offshore HVDC grid is presented in order to improve HVDC cable voltage stability. It is shown that a critical parameter in grid voltage stability is the droop control function, which is (a)

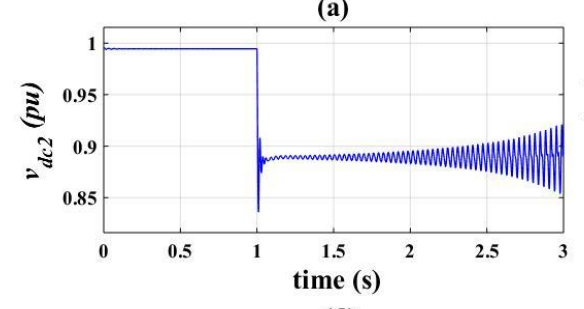

(d)

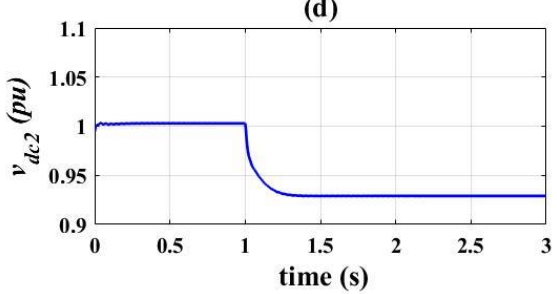

(b)

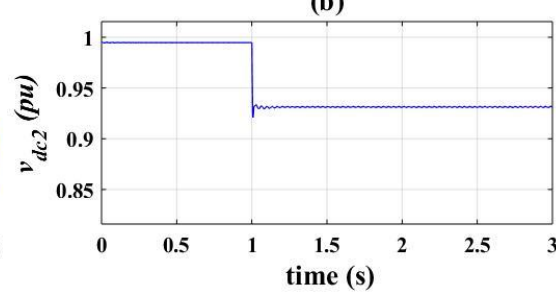

(e)

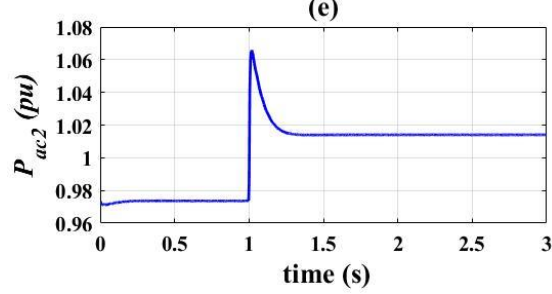

(c)

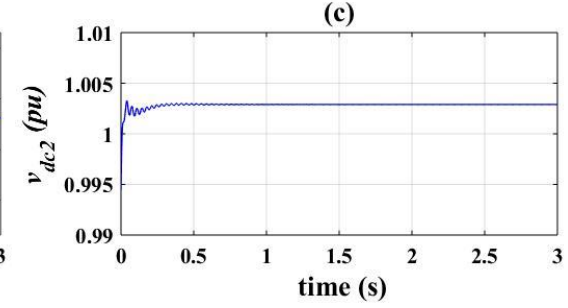

(f)

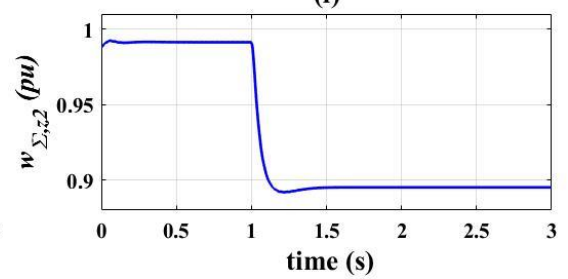

Fig. 9: Simulation results for the time-domain circuit-based model. 
verified through small-signal eigenvalue stability analysis and confirmed by control optimization. The performance of the centralized optimal linear feedback controller is validated via the time-domain circuit-based simulation. It is depicted that the linear feedback controller can improve the grid stability margin both as an extra control layer and as a self-standing droop control function. The DC voltage oscillation index introduced can be used to compare these two scenarios, which shows that COLFC as an extra control layer has better oscillation damping.

\section{References}

[1] J. Beerten, S. D'Arco, and J. A. Suul, "Frequencydependent cable modelling for small-signal stability analysis of VSC-HVDC systems," IET Generation, Transmission \& Distribution, vol. 10, no. 6, pp. 1370-1381, 2016.

[2] A. Lesnicar and R. Marquardt, "An innovative modular multilevel converter topology suitable for a wide power range," in 2003 IEEE Bologna Power Tech Conference Proceedings, 2003, vol. 3, p. 6 pp. Vol.3.

[3] A. Elahidoost and E. Tedeschi, "Expansion of offshore HVDC grids: An overview of contributions, status, challenges and perspectives," in 2017 IEEE 58th International Scientific Conference on Power and Electrical Engineering of Riga Technical University (RTUCON), 2017, pp. 1-7.

[4] D. Connolly, H. Lund, and B. V. Mathiesen, "Smart Energy Europe: The technical and economic impact of one potential $100 \%$ renewable energy scenario for the European Union," Renewable and Sustainable Energy Reviews, vol. 60, pp. 1634-1653, 2016/07/01/ 2016.

[5] J. L. Rueda, W. H. Guaman, J. C. Cepeda, I. Erlich, and A. Vargas, "Hybrid Approach for Power System Operational Planning With Smart Grid and Small-Signal Stability Enhancement Considerations," IEEE Transactions on Smart Grid, vol. 4, no. 1, pp. 530-539, 2013.

[6] A. Elahidoost, L. Furieri, E. Tedeschi, and M. Kamgarpour, "Optimizing HVDC Grid Expansion and Control for Enhancing DC Stability," in 2018 Power Systems Computation Conference (PSCC), 2018, pp. 1-7.

[7] A. Elahidoost, L. Furieri, E. Tedeschi, and M. Kamgarpour, "Reducing HVDC Network Oscillations Considering Wind Intermittency Through Optimized Grid Expansion Decision," in 2018 IEEE Energy Conversion Congress and Exposition (ECCE), 2018, pp. 2683-2690.

[8] W. Wang and M. Barnes, "Power Flow Algorithms for Multi-Terminal VSC-HVDC With Droop Control," IEEE Transactions on Power Systems, vol. 29, no. 4, pp. 17211730, 2014.

[9] G. Pinares and M. Bongiorno, "Analysis and Mitigation of Instabilities Originated From DC-Side Resonances in VSCHVDC Systems," IEEE Transactions on Industry Applications, vol. 52, no. 4, pp. 2807-2815, 2016.

[10] W. Leterme, N. Ahmed, J. Beerten, L. Angquist, D. V. Hertem, and S. Norrga, "A new HVDC grid test system for HVDC grid dynamics and protection studies in EMT-type software," in AC and DC Power Transmission, 11th IET International Conference on, 2015, pp. 1-7.

[11] G. Bergna-Diaz, J. A. Suul, and S. D. Arco, "Energy-Based State-Space Representation of Modular Multilevel Converters with a Constant Equilibrium Point in SteadyState Operation," IEEE Transactions on Power Electronics, vol. 33, no. 6, pp. 4832-4851, 2018.

[12] S. Sanchez, G. Bergna, and E. Tedeschi, "Tuning of control loops for grid-connected Modular Multilevel Converters under a simplified port representation for large system studies," in 2017 Twelfth International Conference on Ecological Vehicles and Renewable Energies (EVER), 2017, pp. 1-8.

[13] P. Kundur, Power System Stability and Control. New York, NY, USA: Mc Graw-Hill, 1994.

[14] A. Antonopoulos, L. Angquist, and H. Nee, "On dynamics and voltage control of the Modular Multilevel Converter," in 2009 13th European Conference on Power Electronics and Applications, 2009, pp. 1-10.

[15] A. Fuchs and M. Morari, "Actuator performance evaluation using LMIs for optimal HVDC placement," in 2013 European Control Conference (ECC), 2013, pp. $1529-1534$.

[16] J. Löfberg, "Yalmip: A toolbox for modeling and optimization in MATLAB," in Proceeding of the CACSD, Taipei, Taiwan, 2004.

[17] K. D. A. E.D. Andersen, "The Mosek Interior Point Optimizer for Linear Programming: An Implementation of the Homogeneous Algorithm. ," in High Performance Optimization., vol. Applied Optimization, vol 33, R. K. In: Frenk H., Terlaky T., Zhang S. (eds), Ed.: Springer, Boston, MA, 2000. 